\title{
Women's preferences for method of abortion and management of miscarriage
}

\author{
Kate Levine, Sharon T Cameron
}

\begin{abstract}
Background and methodology There is growing interest in the UK towards increasing treatment options for women undergoing abortion and miscarriage. Such options include home medical treatment and surgery under local anaesthesia (LA). This study aimed to gauge views of women undergoing abortion and treatment for miscarriage at the Royal Infirmary Edinburgh towards medical treatment at home, and surgery under LA, to determine whether new services should be developed. The study consisted of a self-administered anonymous questionnaire.
\end{abstract}

Results A total of 148 questionnaires were completed by women undergoing a medical abortion ( $n=97 ; 66 \%$ ), surgical abortion $(n=30 ; 20 \%)$ or surgical management of miscarriage ( $n=21 ; 14 \%)$. Women having an abortion expressed a future preference for medical abortion in hospital $(n=64 ; 52 \%)$ at home $(n=31 ; 25 \%)$ or by surgery

\section{Introduction}

Current abortion legislation in Great Britain requires that both mifepristone and misoprostol be administered in an National Health Service (NHS) hospital or approved place. ${ }^{1}$ This does, however, leave the opportunity for women to return home to complete their medical abortion. ${ }^{1}$ Surgical abortion and evacuation of miscarriage in the UK have traditionally been performed under general anaesthesia (GA), but use of local anaesthesia (LA) gives shorter inpatient stay, avoids the risks of GA, and is associated with a lower risk of uterine perforation. ${ }^{2}$ Guidelines from the Royal College of Obstetricians and Gynaecologists recommend that surgical evacuation of the uterus under LA should be available for both induced abortion and miscarriage. 2,3

The Royal Infirmary of Edinburgh (RIE) is the major provider of pregnancy support services for miscarriage and of abortion services in NHS Lothian ( $81 \%$ of abortions in 2007 were performed at RIE; $n=2185$ ). RIE currently offers medical abortion and management of miscarriage in hospital and surgical abortion and management of miscarriage under GA. The principal objective of this study was to determine the preferences of women who underwent induced abortion or miscarriage with regard to possible future treatment options including medical management at home and surgical management under LA.

\section{Methods}

Between November 2006 and January 2007, women undergoing a medical abortion ( $<9$ weeks gestation), surgical abortion ( $\leq 12$ weeks) or management of firsttrimester miscarriage at RIE were invited to complete an

\section{University of Edinburgh, Edinburgh, UK \\ Kate Levine, MBChB, Foundation Year 2 Doctor}

Dean Terrace Centre, Edinburgh, UK

Sharon T Cameron, MD, MRCoG, Consultant Gynaecologist

Correspondence to: Dr Sharon Cameron, Dean Terrace Centre, 18 Dean Terrace, Edinburgh EH4 1NL, UK.

E-mail: Sharon.Cameron@ed.ac.uk under general anaesthesia (GA) $(n=20 ; 17 \%)$ or LA ( $n=$ 7; 6\%). Women having a miscarriage expressed a future preference for surgery under GA $(n=7 ; 35 \%)$, LA ( $n=6$; $30 \%)$ or medical management at home $(n=4 ; 20 \%)$ or in hospital $(n=3 ; 15 \%)$

Conclusions This study shows that medical abortion at home is a potentially popular choice for women having an abortion, with surgical abortion under LA less so. Both home medical management and surgery under LA would appear to be welcome service developments for women needing treatment for a miscarriage.

Keywords acceptability, general anaesthesia, local anaesthesia, medical abortion, miscarriage, surgical abortion

J Fam Pann Reprod Health Care 2009; 35(4): 233-235

(Accepted 6 July 2009)

\section{Key message points}

- As many as one in four women undergoing an induced abortion would choose a home medical abortion if given the choice. This is mainly for reasons of privacy or comfort.

- Home medical management of miscarriage and surgery under local anaesthesia may be welcome new clinical services for women wishing management of miscarriage.

anonymous questionnaire regarding future treatment preferences. The questionnaires were distributed by nursing staff to women on their arrival at RIE for an abortion procedure, or to women with a diagnosis of miscarriage at their clinic visit to arrange subsequent management. The questionnaires contained a brief introduction and explanation of the various treatment methods followed by a series of short questions requiring 'yes/no' answers, 'tick box' responses to statements which corresponded most closely to patients' views and openended questions. Limited demographic data were collected including age and postcode, which were used to obtain a Carstairs deprivation category score ${ }^{4}$ Women were asked to place completed questionnaires in a collection box in a sealed opaque envelope. Women undergoing abortion thus completed questionnaires immediately prior to their medical or surgical procedure, whereas women undergoing miscarriage completed them on the day that they decided on subsequent management.

\section{Statistical analysis}

Statistical analysis was performed on coded data using Statistical Package for the Social Sciences (SPSS) software (SPSS Inc., Chicago, IL, USA). Comparisons were carried out using the Pearson Chi-square test or Fisher's exact test. Statistical significance was defined as $p<0.05$. The study was conducted as part of the first author's medical student specialist project.

\section{Ethical approval}

The ethics committee responsible for medical students reviewed the study proposal and concluded that regional ethical committee approval was not required. 


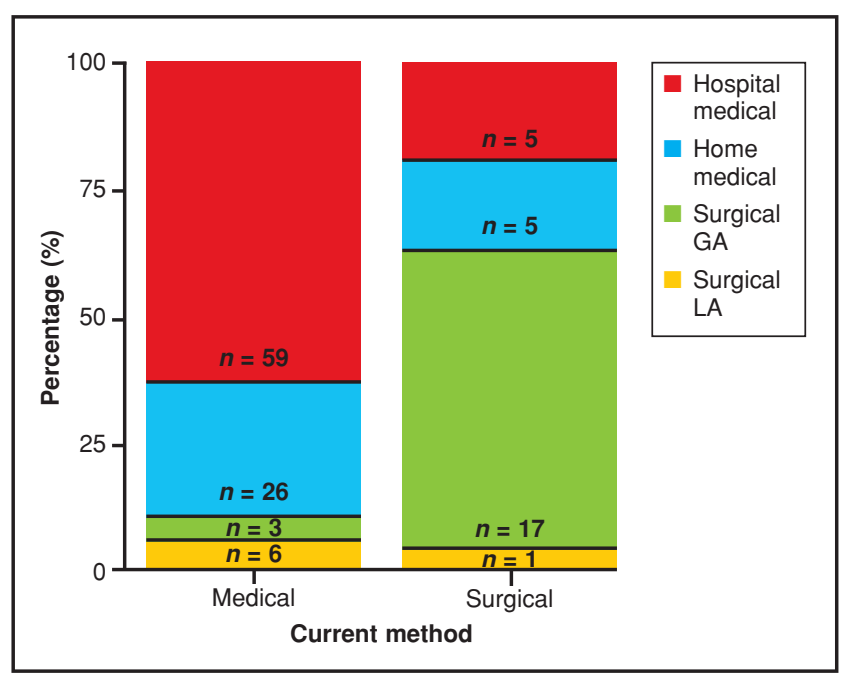

Figure 1 Preferred future choice of women undergoing medical and surgical abortion, by current method. GA, general anaesthesia; LA, local anaesthesia

\section{Results}

Questionnaires were distributed to 100 women choosing medical abortion, 60 choosing surgical abortion and 60 women with a miscarriage. A total of 148 completed questionnaires were returned comprising 97, 30 and 21 from medical abortion, surgical abortion and miscarriage groups, respectively (completion rates of $97 \%, 50 \%$ and $35 \%$, respectively). There was no significant difference in the mean age of respondents in each group, although women having a miscarriage tended to be older $(23,21$ vs 34 years from medical abortion, surgical abortion and miscarriage groups, respectively). There was no significant difference in deprivation category scores between groups (data not shown).

\section{Preference of women undergoing abortion}

All 97 women undergoing a medical abortion stated that they had chosen this method, but only $50 \%$ of respondents undergoing surgical abortion $(n=15)$ had chosen surgery, with the remainder stating that they had not been offered a choice of method (in most cases because they had presented too late for medical abortion). When asked which abortion method women would choose in the future, if given the choice of medical at home or in hospital, or surgical under GA or LA, the majority of women $(88 \% ; n$ $=87$ ) undergoing a medical abortion stated that they would choose a medical method again, either in hospital (61\%; $n$ $=59)$ or at home $(27 \% ; \quad n=26)$. Only $4 \%(n=4)$ stated that they would opt for surgical abortion under GA and $8 \%$ $(n=8)$ for surgery under LA (Figure 1).

The majority $(18 / 30 ; 60 \%)$ of women currently having a surgical abortion expressed a future preference for this method again, with only $6 \%(n=1)$ opting to have this performed under LA (Figure 1). Table 1 shows the first and second future choices if all methods were available.

When asked which type of anaesthesia women would choose if they had no choice of method, but had to have a surgical abortion, most women $(n=93,73 \%)$ stated that they would prefer GA. The commonest reasons for this were because of their desire to be asleep and unaware $(n=$ $82 ; 88 \%)$, a fear of pain $(n=7 ; 8 \%)$ and the avoidance of upsetting memories $(n=4 ; 4 \%)$. The remaining $27 \%(n=$ 34 ) of women who would choose LA stated a desire to be awake and aware $(n=17 ; 50 \%)$, the avoidance of side effects of GA $(n=11 ; 33 \%)$, a quicker recovery $(n=4$; $10 \%)$ and current health problems $(n=2 ; 7 \%)$. Of the women preferring LA, five women were currently having a surgical abortion $(5 / 28 ; 18 \%)$ and 27 were from the medical abortion group $(27 / 96 ; 28 \%)$. This was not statistically significant $(p=0.67)$.

When asked which setting (hospital or home) women would choose if they had no choice of method, but had to have a medical abortion, the majority $(n=88 ; 69 \%)$ would choose hospital. The main reasons were concern about adverse events at home/perceived increase in safety within hospital $(n=42 ; 48 \%)$ together with the "presence of professional support' $(n=22 ; 25 \%)$. Other reasons included pain relief $(n=15 ; 17 \%)$, reassurance that the pregnancy had passed $(n=7 ; 8 \%)$ and confidentiality $(n=2 ; 2 \%)$. Of the $31 \%(n=39)$ of women who would choose a home setting, this was for reasons of privacy or comfort $(n=26 ; 67 \%)$, family being present $(n=8 ; 21 \%)$, the ability to carry out daily tasks whilst at home $(n=2$; $4 \%)$, fewer hospital visits $(n=2 ; 4 \%)$ and the 'shame of the procedure' $(n=2 ; 4 \%)$. Of those women who would prefer a home setting, five women were currently having a surgical abortion $(5 / 28 ; 18 \%)$ and 33 were from the medical abortion group $(33 / 97 ; 34 \%)$. These results were not statistically different $(p=0.089)$.

\section{Preference of women having a miscarriage}

All 21 respondents had chosen surgical evacuation for managing their current miscarriage. The most popular future choice was surgery under GA $(n=7 ; 35 \%)$ (Table 1$)$. The commonest reasons for choosing surgery under GA mirrored those reasons given by those having an induced abortion (results not shown). In response to their choice of anaesthesia if only the surgical method were offered, $35 \%$ $(n=7)$ would choose LA. Regarding choice of setting if only medical methods were available, $67 \%$ of respondents $(n=14)$ would opt for hospital management with $33 \%(n=7)$ choosing the home setting. The commonest reasons for choosing hospital and home settings mirrored those reasons given by women having an induced abortion (results not shown).

\section{Influential factors on method}

In combining responses from women in all groups, there was a (non-significant) tendency for older age groups (>36 years) to be more accepting of LA $(p=0.061)$. There was

Table 1 Preferred future first and second choice of method by women undergoing induced abortion $(n=127)$ and miscarriage $(n=21)$. Women did not always express both a first and second choice

\begin{tabular}{lllll}
\hline Abortion method & $\begin{array}{l}\text { Abortion first choice } \\
(\boldsymbol{n}=\mathbf{1 2 2})[\boldsymbol{n}(\%)]\end{array}$ & $\begin{array}{l}\text { Abortion second choice } \\
(\boldsymbol{n}=\mathbf{9 9})[\boldsymbol{n}(\%)]\end{array}$ & $\begin{array}{l}\text { Miscarriage first choice } \\
(\boldsymbol{n}=\mathbf{2 0})[\boldsymbol{n}(\%)]\end{array}$ & $\begin{array}{l}\text { Miscarriage second choice } \\
(\boldsymbol{n}=\mathbf{1 4})[\boldsymbol{n}(\%)]\end{array}$ \\
\hline Hospital medical & $64(52)$ & $35(35)$ & $3(15)$ & $3(21)$ \\
Home medical & $31(25)$ & $29(29)$ & $4(20)$ & 1 \\
Surgical GA & $20(17)$ & $21(21)$ & $7(35)$ & $5(36)$ \\
Surgical LA & $7(6)$ & $14(14)$ & $6(30)$ & $5(36)$ \\
\hline
\end{tabular}

GA, general anaesthesia; LA, local anaesthesia. 
no association between choice of anaesthesia and deprivation category score (results not shown).

\section{Discussion}

This is the first study to determine women's views on four possible ways of managing miscarriage or induced abortion. This study showed that if women in Lothian having a medical abortion were offered all four options in the future, the hospital medical method would be the most popular future choice. Clearly the majority of respondents were women currently choosing a medical abortion, which is likely to influence the choice of method overall. Also, women choosing medical abortion had the highest response rate, which may reflect the duration of time that they remained in hospital and thus had available time to complete the questionnaire. Nevertheless, medical abortion at home was the preferred option for almost one in four women having a medical abortion. Although this cannot legally be initiated at home, our results suggest that allowing women to leave our medical abortion service soon after administration of misoprostol and to subsequently abort at home could be a welcome service development.5,6 A recent evaluation of different sites for early medical abortion in England reported that the majority of women treated as outpatients were satisfied with this method. ${ }^{6}$ Furthermore, one pilot of early medical abortion on this 'outpatient' basis reported that it was significantly cheaper for the NHS than providing an inpatient service. 5

In our study, only a minority $(6 \%)$ of those undergoing a surgical abortion stated that they would opt for this under LA. This may be because women in our population have tended to choose surgical abortion because they want to be asleep and unaware of the procedure. ${ }^{7}$ Nevertheless, our study suggests that surgery under LA would be a welcome development for managing miscarriage, since almost one in three women in our miscarriage group stated that this would be their future method of choice. There was also good support for home medical management of miscarriage. Clearly, however, the limited numbers in this group mean that the precise extent of support cannot be accurately determined.

\section{Conclusions}

Our study suggests that one quarter of women undergoing an early medical abortion in our hospital service would choose to abort at home if this were possible. Allowing women to go home soon after they have received misoprostol may therefore offer a welcome service to women and be less costly to the NHS whilst remaining within the current legal framework. Women undergoing management of a miscarriage (although few in number) were also keen to opt for the new choices of home medical management and surgery under LA. By improving patient choice, these new services could help improve women's journeys through difficult life events such as abortion or miscarriage.

\section{Acknowledgements}

The authors would like to thank the nursing staff of Bruntsfield Suite, Day Case Gynaecology Surgery and Pregnancy Support, Royal Infirmary Edinburgh, for distributing and collecting questionnaires.

\section{Statements on funding and competing interests \\ Funding None identified.}

Competing interests None identified.

References

1 British Medical Association. First Trimester Abortion. A Briefing Paper by the BMA's Medical Ethics Committee. ARM 2007. http://www.bma.org.uk/images/Firsttrimesterabortion_tcm41146722.pdf [Accessed 1 June 2009].

2 Royal College of Obstetricians and Gynaecologists. The Care of Women Requesting Induced Abortion (Evidence-based Clinical Guideline No. 7.) September 2004. http://www.rcog. org.uk/womens-health/clinical-guidance/care-womenrequesting-induced-abortion [Accessed 1 June 2009].

3 Royal College of Obstetricians and Gynaecologists. The Management of Early Pregnancy Loss (Green-top Guideline No. 25). October 2006. http://www.rcog.org.uk/womenshealth/clinical-guidance/management-early-pregnancy-lossgreen-top-25 [Accessed 1 June 2009].

4 McLoone P. Carstairs Scores for Scottish Postcode Sectors from the 2001 Census - Report. March 2004. http://www. sphsu.mrc.ac.uk/sitepage.php?page=carstairs [Accessed 1 June 2009].

5 Tupper C, Andrews J. Setting up an outpatient service for early medical termination. J Fam Plan Reprod Health Care 2007; 33: 199-202.

6 Department of Health. Evaluation of Early Medical Abortion (EMA) Pilot Sites. May 2008. http://www.dh.gov.uk/en/ Publicationsandstatistics/Publications/PublicationsPolicyAnd Guidance/DH_084618 [Accessed 1 June 2009].

7 Cameron ST, Glasier A, Logan J, Benton L, Baird DT. Impact of the introduction of new medical methods on therapeutic abortions at the Royal Infirmary of Edinburgh. $\mathrm{Br} \mathrm{J}$ Obstet Gynaecol 1996; 103: 1222-1229.
The Complete Guide to IVF: An Inside View of Fertility Clinics and Treatment. Kate Brian. London, UK: Piatkus, 2009. ISBN-13: 978-07499-0970-3. Price: £12.99. Pages: 304 (paperback)

This is an excellent book, written by an ex-patient and an expert. The book is aimed at those couples that find themselves in the position of going for in vitro fertilisation (IVF) treatment. It is clearly written, systematic and balanced. In a field where there are often conflicting views and practices, this book provides a carefully researched, impartial guide for couples. I strongly recommend it to all patients who are contemplating IVF treatment.

As I am sure that this book will be updated and revised in the years to come, I take this opportunity to offer some suggestions for future editions. I think one of the most difficult situations that couples find themselves in is not so much when they don't get pregnant following treatment, but more so when things go wrong in the clinic. Most of us can cope with the ups and downs of life, but we all want to feel we have had the best treatment that can possibly be offered. think, therefore, that it would be helpful to have a section entitled "When things go wrong" containing advice on to how to proceed specifically for those couples that attend for clinic appointments but who are unhappy with their experience. Another area that I think is worth exploring in a little more depth in this multicultural society in which we live are the pressures and challenges faced by couples from different ethnic and cultural backgrounds Certainly couples from the Indian subcontinen face a number of challenges - be they cultural or religious - which many find difficult to overcome. Lastly, it might be helpful to expand the section on preparation before attending for fertility treatment to include topics such as being checked for rubella immunity, folic acid (this is mentioned but there are certain categories where the woman should be on a higher dose) and the woman being up to date with cervical smears. There is also a requirement for viral screening prior to treatment, and again it would be helpful to have this explained.

Whilst this book is aimed squarely at the patient population, there is one section describing the waiting room experience of patients that I think is an absolute 'must read' for all clinic staff. I am sure that we all recognise this particular experience.

Reviewed by Masoud Afnan, FRCOG

Consultant Obstetrician and Gynaecologist and Fertility Specialist, Birmingham Women's Hospital Foundation NHS Trust, Birmingham, UK 